\title{
Article \\ Platelet Toll-like Receptor 4-Related Innate Immunity Potentially Participates in Transfusion Reactions Independent of ABO Compatibility: An Ex Vivo Study
}

\author{
Chien-Sung Tsai ${ }^{1,2,3}$, Mei-Hua Hu ${ }^{4,5}$, Yung-Chi Hsu 6 (D) and Go-Shine Huang ${ }^{6, *}$ \\ 1 Department of Surgery, Division of Cardiovascular Surgery, Tri-Service General Hospital, Taipei 114, Taiwan; \\ sung1500@mail.ndmctsgh.edu.tw \\ 2 Department and Graduate Institute of Pharmacology, National Defense Medical Center, Taipei 114, Taiwan \\ 3 Institute of Pharmacy, I.M. Sechenov First Moscow State Medical University, Moscow 119991, Russia \\ 4 Department of Pediatrics, Division of Pediatric General Medicine, Chang Gung Memorial Hospital at Linkou, \\ College of Medicine, Chang Gung University, Taoyuan 333, Taiwan; p65952@gmail.com \\ 5 Graduate Institute of Clinical Medical Sciences, College of Medicine, Chang Gung University, \\ Taoyuan 333, Taiwan \\ 6 National Defense Medical Center, Department of Anesthesiology, Tri-Service General Hospital, Taipei 114, \\ Taiwan; X0939778570@gmail.com \\ * Correspondence: kshgodoc@gmail.com; Tel.: +886-(2)-8792-7128; Fax: +886-(2)-8792-7127
}

check for updates

Citation: Tsai, C.-S.; Hu, M.-H.; Hsu, Y.-C.; Huang, G.-S. Platelet Toll-like Receptor 4-Related Innate Immunity Potentially Participates in Transfusion Reactions Independent of ABO Compatibility: An Ex Vivo Study. Biomedicines 2022, 10, 29 https://doi.org/10.3390/ biomedicines 10010029

Academic Editor: Juan Sahuquillo

Received: 16 November 2021

Accepted: 20 December 2021

Published: 23 December 2021

Publisher's Note: MDPI stays neutral with regard to jurisdictional claims in published maps and institutional affiliations.

Copyright: (c) 2021 by the authors. Licensee MDPI, Basel, Switzerland. This article is an open access article distributed under the terms and conditions of the Creative Commons Attribution (CC BY) license (https:// creativecommons.org/licenses/by/ $4.0 /)$.

\begin{abstract}
The role of platelet TLR4 in transfusion reactions remains unclear. This study analyzed platelet TLR4 and certain damage-associated molecular patterns (DAMPs) and evaluated how ABO compatibility affected TLR4 expression after a simulated ex vivo transfusion. A blood bank was the source of donor red blood cells. Blood from patients undergoing cardiac surgery was processed to generate a washed platelet suspension to which the donor blood was added in concentrations 1 , 5 , and $10 \%(v / v)$. Blood-mixing experiments were performed on four groups: a $0.9 \%$ saline control group ( $n=31$ ); a matched-blood-type mixing group (group $\mathrm{M}, n=20$ ); an uncross-matched ABOspecific mixing group (group $\mathrm{S}, n=20$ ); and an ABO-incompatible blood mixing group (group I, $n=20$ ). TLR4 expression in the platelets was determined after blood mixing. We evaluated levels of TLR4-binding DAMPs (HMGB1, S100A8, S100A9, and SAA), lipopolysaccharide-binding protein, and endpoint proteins in the TLR4 signaling pathway. In the M, S, and I groups, 1, 5, and 10\% blood mixtures significantly increased TLR4 expression (all $p<0.001$ ) in a concentration-dependent manner. Groups M, S, and I were not discovered to have significantly differing TLR4 expression $(p=0.148)$. HMGB1, S100A8, and S100A9 levels were elevated in response to blood mixing, but SAA, lipopolysaccharide-binding protein, TNF- $\alpha$, IL- $1 \beta$, and IL- 6 levels were not. Blood mixing may elicit innate immune responses by upregulating platelet TLR4 and DAMPs unassociated with ABO compatibility, suggesting that innate immunity through TLR4-mediated signaling may induce transfusion reactions.
\end{abstract}

Keywords: toll-like receptor 4; innate immunity; platelet; blood mixing; transfusion reaction

\section{Introduction}

Blood transfusions are critical interventions, particularly for patients undergoing hemorrhagic shock. However, despite significant advancements in the safety of blood transfusions, such procedures are still associated with major risks. During blood transfusions, the mechanisms of immune reactions, specifically innate immune reactions, are unclear. Related reactions can result from transfusion with cross-matched blood, uncrossmatched type-specific blood, or ABO-incompatible blood [1]. Immune reactions to blood transfusions involve complex interactions among various soluble factors and immune cells, including platelets [1].

Platelets have a major role in thrombosis and hemostasis and are known to have a mediating effect on the body's innate immune response [2]. They upregulate Toll-like 
receptor 4 (TLR4), serve as sentinels in the immune system, and are essential for stimulating adaptive immune responses [2-7]. We hypothesized that blood transfusion could induce platelet-mediated innate immune reactions caused by the interaction between TLR4 and damage-associated molecular patterns (DAMPs) [8]. Thus, we aim to evaluate the levels of TLR4, lipopolysaccharide (LPS)-binding protein, and certain DAMPs, such as S100 calcium-binding protein A8 (calgranulin A, S100A8, and MRP8), high mobility group box 1 (HMGB1), serum amyloid A (SAA), and S100 calcium-binding protein A9 (S100A9, MRP14, and calgranulin B), in a simulated cross-reaction ex vivo. Furthermore, proinflammatory cytokines downstream of TLR4 signaling, such as proinflammatory cytokines (IL-6, TNF- $\alpha$, and IL-1 $\beta$ ), were evaluated [8]. This study might be valuable since it is the leading study to evaluate the relationship between transfusion and innate immunity through TLR4 bind with DAMPs then test signal transduction.

\section{Material and Methods}

\subsection{Reagents and Flow Cytometry}

The antibodies employed were anti-TLR4-PE antibody (BD Biosciences, Franklin Lakes, NJ, USA), a monoclonal antibody targeting TLR4 expressed on the platelet cell surface; and anti-CD41a-FITC (BD Biosciences), which is a platelet-specific monoclonal antibody that, without needing to be activated, recognizes the platelet GPIIb/IIIa complex. Nonspecific binding was investigated using IgG1 k-FITC and IgG1 k-PE antibodies (BD Biosciences). Platelets were stimulated using thrombin (Sigma, St. Louis, MO, USA). In our laboratory, we prepared the $\mathrm{pH} 7.4$ platelet wash buffer $(20 \mathrm{mM} \mathrm{HEPES}, 145 \mathrm{mM} \mathrm{NaCl}$, and $9 \mathrm{mM} \mathrm{Na} 2$ EDTA) and pH 7.4 HEPES-buffered Tyrode's solution (10 mM HEPES, $1.61 \mathrm{mM}$ $\mathrm{KCl}, 0.42 \mathrm{mM} \mathrm{Na}_{2} \mathrm{HPO}_{4}, 11.9 \mathrm{mM} \mathrm{NaHCO} 3,136.89 \mathrm{mM} \mathrm{NaCl}, 1.05 \mathrm{mM} \mathrm{MgCl}$, and $5.6 \mathrm{mM}$ glucose).

\subsection{Blood Sampling}

Approval for this research was granted by the Tri-Service General Hospital Institutional Review Board (TSGHIRB 1-102-05-014, TSGHIRB 1-107-05-015), and before enrollment, all participants provided written informed consent. Rh-negative patients were excluded from this study. Red blood cells (unwashed) with a 55-60\% hematocrit result were obtained from our hospital blood bank and stored at $2-4{ }^{\circ} \mathrm{C}$. We obtained blood samples from an arterial catheter before anesthesia induction in individuals scheduled to undergo cardiac surgery. Each sample was treated with an anticoagulant, which consisted of a 1:9 volume of $3.8 \%$ sodium citrate solution. Blood transfusions were simulated ex vivo through blood mixing. Patients were divided into four groups in terms of blood mixing reactions: a $0.9 \%$ saline control group, a matched-blood-type mixing group (group $\mathrm{M}$ ), an uncross-matched $\mathrm{ABO}$-specific mixing group (group $\mathrm{S}$ ), and an $\mathrm{ABO}$-incompatible mixing group (group I).

The primary outcome measures were TLR4 expression differences among three groups (I, S, and M) and the influence of blood mixing on platelet TLR4 expression. The secondary outcome measures were the influence of blood mixing on the levels of LPS-binding protein and DAMPs, including HMGB1, SAA, S100A8, and S100A9. Furthermore, the levels of molecules downstream of TLR4 signaling were evaluated in each of the three groups (I, S, and $\mathrm{M})[8]$.

\subsection{Flow Cytometry Analysis of TLR4 Expression}

Whole blood underwent centrifugation at $37^{\circ} \mathrm{C}$ for $10 \mathrm{~min}$ at $200 \times \mathrm{g}$. We collected the upper phase (plasma) carefully, and we disposed of the interphase (buffy coat chiefly comprising leukocytes and some platelets) and lower phase (red cells). Subsequently, the upper phase was subjected to centrifugation $(10 \mathrm{~min}$ at $2000 \times \mathrm{g})$, and buffer was employed to gently resuspend the resultant pellet for washing platelets. For preparing washed platelets, we subjected the suspension to centrifugation $(10 \mathrm{~min}$ at $2000 \times \mathrm{g})$ and resuspended the platelets in HEPES-buffered Tyrode's solution. Subsequently, the suspension was altered 
so that the final number of platelets was $150,000 / \mu \mathrm{L}$ to $450,000 / \mu \mathrm{L}$. Thereafter, the washed platelets without or with $0.9 \%$ saline added at 1,5 , or $10 \%(v / v)$ were used as controls. For blood mixing reactions, donor red blood cells were immediately mixed with the washed platelets at 1,5 , or $10 \%(v / v)$ and incubated at $37^{\circ} \mathrm{C}$ for $5 \mathrm{~min}$.

\subsection{Quantification of TLR4 Expression in Washed Platelets}

We investigated platelet TLR4 expression in mixed samples in the presence of thrombin (final concentration: $0.2 \mathrm{U} / \mathrm{mL}$ ) by incubating the samples at an ambient temperature of $23-26^{\circ} \mathrm{C}$ for $5 \mathrm{~min}$. Thrombin is generated during tissue injury, such as that sustained during cardiac surgery [9]. It is a key component of the blood coagulation cascade and a potent platelet stimulator. Thrombin is commonly used in sample preparation protocols for platelet analysis. To quantify the TLR4 expression, sample staining was conducted using a saturating concentration of anti-CD41a-FITC and anti-TLR4-PE monoclonal antibodies, and the samples were incubated for $20 \mathrm{~min}$ in a dark environment at $22-26{ }^{\circ} \mathrm{C}$. Subsequently, before they underwent flow cytometry analysis, the samples were fixed using paraformaldehyde $(1 \%)$ for $30 \mathrm{~min}$ at a temperature of $4{ }^{\circ} \mathrm{C}$. PE-labeled IgG1 $\mathrm{K}$ and FITClabeled IgG1K functioned as background controls. We performed identification of each platelet by using side scattering (granularity features) and anti-CD41a-FITC immunofluorescence on a logarithmic-scaled dot plot. This article presents the results as the mean fluorescence intensity (MFI) of TLR4-PE expression, and for each sample, we gathered reads from 10,000 platelets.

\subsection{ELISA Analysis}

We assessed the levels of LPS-binding protein and of certain DAMPs that may interact with TLR4 after blood mixing. Donor red blood cells were immediately added to whole blood from recipients at 1,5 , or $10 \%(v / v)$ and incubated for $30 \mathrm{~min}$ at $37^{\circ} \mathrm{C}$. The mixed blood then underwent centrifugation $\left(10 \mathrm{~min}, 37^{\circ} \mathrm{C}, 100 \times \mathrm{g}\right)$. We carefully gathered the upper phase (plasma), but we disposed of the interphase (buffy coat chiefly comprising leukocytes and some platelets) and lower phase (red cells). The plasma was analyzed using ELISA kits (in accordance with manufacturer instructions) to determine the concentrations of HMGB1 (Aviva Systems Biology, San Diego, CA, USA), S100A8 (Circulex, MBL, Nagano, Japan), S100A9 (Circulex, MBL), SAA (Abnova Co., Taipei, Taiwan), and LPS-binding protein (Aviva Systems Biology).

\subsection{Multiplex Immunoassays}

The amounts of proinflammatory cytokines IL- 6 , TNF- $\alpha$, and IL- $1 \beta$ in the plasma samples were determined using the ProcartaPlex Multiplex Immunoassay (Affymetrix eBioscience, Thermo Fisher Scientific, Waltham, MA, USA) by following the manufacturer's instructions.

\subsection{Statistical Analysis}

One-way ANOVA was performed for a comparison of the four groups' demographic variables (control, I, M, and S groups). Furthermore, ANOVA was performed to compare the levels of TLR4, LPS-binding protein, DAMPs (e.g., HMGB1, S100A8, S100A9, and SAA), and TLR4-regulated cytokines (e.g., IL-6, TNF- $\alpha$, and IL-1 $\beta$ ) in each of three groups (M, S, and I), with Scheffé's post hoc test then performed. Two-way ANOVA was employed to identify differences between three groups (I, M, and S) over various concentrations. All tests were two-sided, and statistical significance was indicated by $p<0.05$. SPSS (version 20; SPSS, Inc., Chicago, IL, USA) was used for all analyses.

\section{Results}

Demographic characteristics did not differ significantly among the four groups (Table 1). Regarding platelet TLR4 expression, the flow cytometric analysis findings are displayed in Supplemental Figure S1 as fluorescence dot plots showing TLR4 expression in isotype 
controls (Supplemental Figure S1A ), unstimulated platelets (Supplemental Figure S1B), and platelets stimulated using thrombin not mixed with (Supplemental Figure S1C) and mixed with 10\% (Supplemental Figure S1D) donor blood. The histograms for these latter three are overlaid in Supplemental Figure S1E.

Table 1. Demographic characteristics of recipients in $0.9 \%$ saline control and groups $\mathrm{M}$, S, and I.

\begin{tabular}{cccccc}
\hline Groups & $\begin{array}{c}\mathbf{0 . 9 \%} \text { Saline } \\
\text { Control }\end{array}$ & M & S & I & $\begin{array}{c}p \text { Value of } \\
\text { Four Groups }\end{array}$ \\
\hline Number of cases & 31 & 20 & 20 & 20 & \\
\hline Age (years) & $60.4 \pm 12.0$ & $61.2 \pm 10.7$ & $60.1 \pm 11.7$ & $65.7 \pm 13.7$ & 0.424 \\
\hline Height $(\mathrm{cm})$ & $165.0 \pm 7.9$ & $165.1 \pm 8.2$ & $165.1 \pm 7.3$ & $165.8 \pm 8.2$ & 0.991 \\
\hline Weight $(\mathrm{kg})$ & $67.7 \pm 11.9$ & $69.1 \pm 13.3$ & $66.1 \pm 10.8$ & $65.3 \pm 9.2$ & 0.724 \\
\hline $\begin{array}{c}\text { Body mass index } \\
\left(\mathrm{kg} / \mathrm{m}^{2}\right)\end{array}$ & $24.9 \pm 4.2$ & $25.2 \pm 4.1$ & $24.2 \pm 3.5$ & $23.8 \pm 3.4$ & 0.634 \\
\hline Women/Men & $12 / 19$ & $4 / 16$ & $6 / 14$ & $7 / 13$ & 0.497
\end{tabular}

Data are presented as the mean \pm SD. M, matched blood type mixture; group S, uncross-matched ABO groupspecific mixture; I, ABO incompatibility blood mixture.

\subsection{Effect of TLR4 Expression after the Addition of $0.9 \%$ Saline}

We examined the control groups to reveal whether TLR4 expression levels could be determined in blood-mixing experiments by using a washed platelet mixed with $0.9 \%$ saline $(1,5$, and $10 \%(v / v))$. The TLR4 expression levels did not differ significantly following stimulation with thrombin or the $0.9 \%$ saline $(1,5$, and $10 \%(v / v) ; p=0.892$, Figure 1$)$.

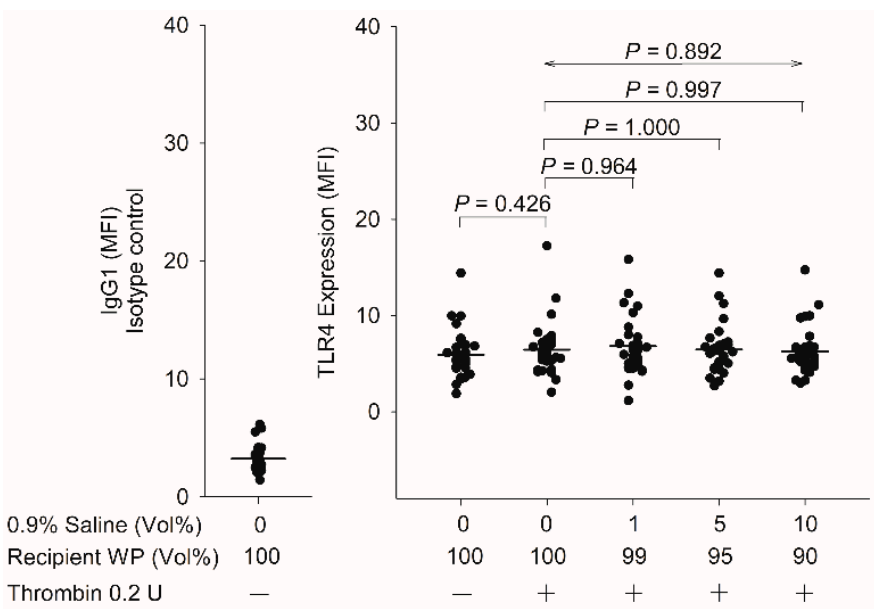

Figure 1. Effect of TLR4 expression after $0.9 \%$ saline was mixed with washed platelets (group $\mathrm{M}, n=10$; group $\mathrm{S}, n=10$; and group I, $n=11$ ). WP, washed platelets; vol, volume; MFI, mean fluorescence intensity. Data are presented as mean \pm SD.

\subsection{Effect of TLR4 Expression after the Addition of Donor Red Blood Cells}

The 1,5 , and $10 \%(v / v)$ blood mixtures protocols induced a significant increase in TLR4 expression levels in groups I, M, and S (all $p<0.001$; Figure 2), and the increase was concentration dependent. Compared with the control level, TLR4 expression was increased by 147,288 , and $381 \%$ after 1,5 , and $10 \%$ blood mixing, respectively $(n=60, p<0.001$; Figure 2). In group M, TLR4 expression increased by 143, 308, and 399\% after 1, 5, and 10\% mixing, respectively, compared with the control level $(n=20, p<0.001$; Figure 2), whereas the increases in group S were 157, 291, and 380\% $(n=20, p<0.001$; Figure 2$)$ and in group I were 142, 265, and 362\%, respectively $(n=20, p<0.001$; Figure 2$)$. However, the three 
experimental groups' TLR4 expression levels were not significantly different $(p=0.148$; Figure 2).
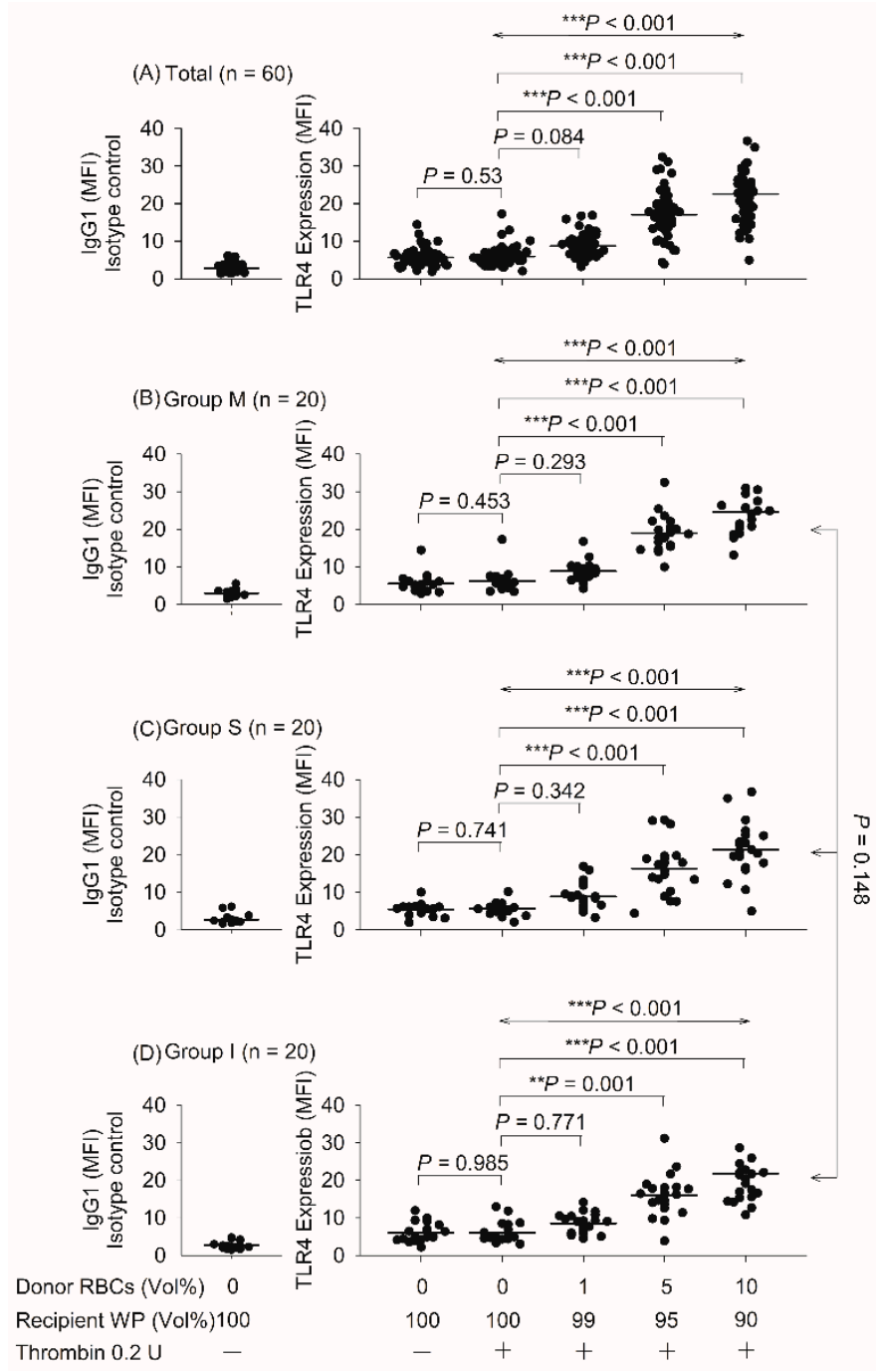

Figure 2. Effect of TLR4 expression on groups M, S, and I after the mixing of donor red blood cells and washed platelets. MFI, mean fluorescence intensity; RBCs, red blood cells; vol, volume; WP, washed platelets. Data are expressed as mean \pm SD. ${ }^{* *} p<0.01,{ }^{* * *} p<0.001$.

\subsection{Effect of DAMPs and LPS-Binding Protein in Plasma}

HMGB1, S100A8, and S100A9 levels were significantly increased after blood mixing (Figure 3). Compared with the control level, the total HMGB1 levels were significantly increased by 122,200 , and $272 \%$ following 1,5 , and $10 \%$ mixing, respectively $(p<0.001$; Figure 3). Total S100A8 levels were 104, 114, and 123\% higher ( $p<0.001$; Figure 3 ) and total S100A9 levels 115, 138, and 150\% higher, respectively ( $p=0.012$; Figure 3$)$. However, total SAA levels were not significantly increased in any of the three experimental groups (I, M, and S: $p=0.988,0.588$, and 0.999, respectively; Figure 3). 


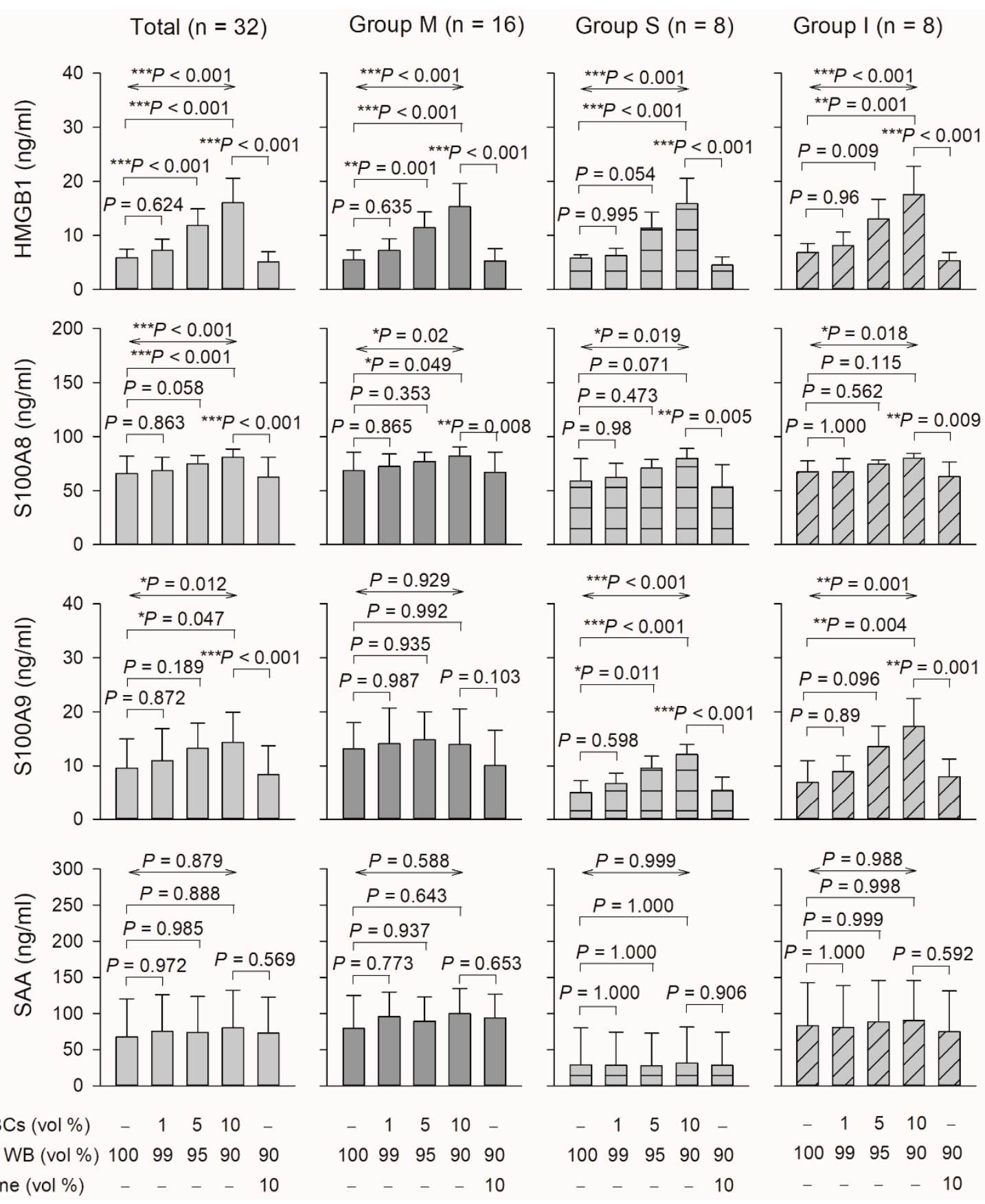

Figure 3. Effect of DAMPs (HMGB1, S100A8, S100A9, and SAA) in the plasma prepared from mixing donor red blood cells and recipient whole blood on total blood mixing and groups $\mathrm{M}$, $\mathrm{S}$, and $\mathrm{I}$. HMGB1, high mobility group box-1; SAA, serum amyloid A; RBCs, red blood cells; vol, volume; WP, washed platelets. Data are expressed as mean \pm SD. ${ }^{*} p<0.05,{ }^{* *} p<0.01,{ }^{* * *} p<0.001$.

\subsection{Effect of Proinflammatory Cytokines Downstream of the TLR4 Signaling Pathway in Plasma}

Levels of total LPS-binding protein involved in TLR4 signaling $(p=0.526)$ and endpoint proteins, namely total TNF- $\alpha$, IL-1 $\beta$, and IL-6 ( $p=0.998,0.806$, and 0.87 , respectively), were not elevated after blood mixing (Figure 4). 


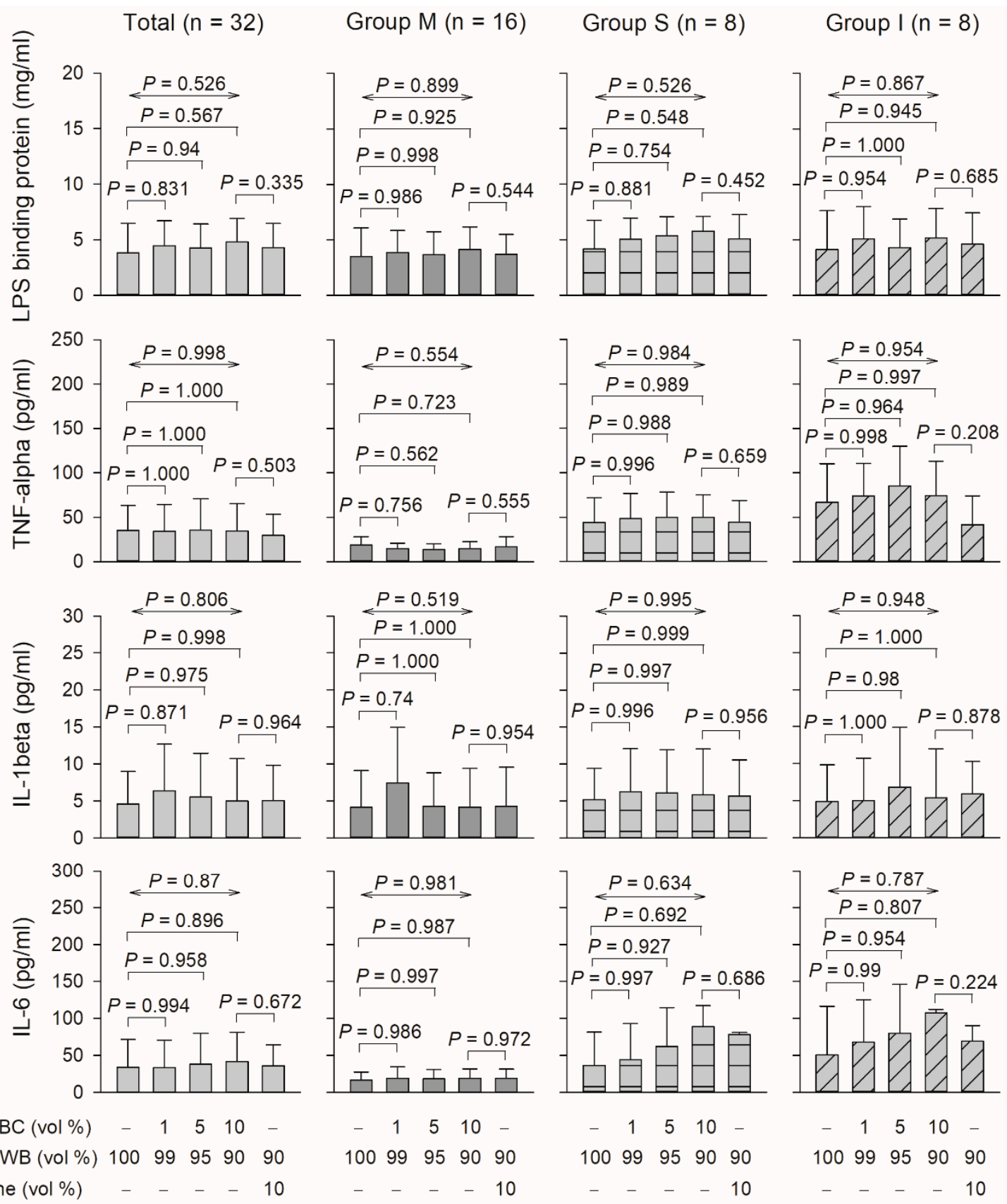

Figure 4. Effect of LPS-binding protein and proinflammatory cytokines in plasma prepared from mixing donor red blood cells and recipient whole blood on groups M, S, and I. RBCs, red blood cells; vol, volume; WP, washed platelets. Data are expressed as mean \pm SD.

\section{Discussion}

This study revealed that after ex vivo blood mixing, TLR4 expression was upregulated in platelets in groups M, S, and I. Besides its important role in thrombosis and hemostasis, platelet TLR4 makes a major contribution to increasing immune and inflammatory responses [2]. This may also be true for platelet TLR4-related immune and inflammatory responses after a transfusion [2]. Platelet TLR4 expression influences innate immunity $[5,7,8,10,11]$, leading to an adaptive immune response [3-7] and inflammation [12], as noted in transfusion reactions. Thus, platelet TLR4 may be a pathophysiological link between innate immunity and transfusion reactions. 
TLR4 recognizes DAMPs [13], which are associated with host cell components released after cell damage $[5,14,15]$. We revealed that levels of certain DAMPs, including HMGB1, S100A8, and S100A9, were elevated after blood mixing, and this finding was corroborated by other reports [16-20] on blood transfusions (Figure 3). For example, first, a transfusion of red blood cells increases vulnerability to lung inflammation through HMGB1 release and induces lung endothelial cell necroptosis [19]. Second, stored human red blood cells contain soluble HMGB1, the levels of which are elevated during storage [16]. Third, salvaged blood analyses identified sustained high levels of certain DAMPs, including S100A8 and S100A9. Transfusion reactions may result from increased levels of TLR4 and certain DAMPs, including HMGB1, S100A8, and S100A9, that bind to TLR4 (Figure 3). The upregulation of TLR4 and certain DAMPs suggests that innate immunity conferred through TLR4-mediated signaling can induce a transfusion reaction.

Upregulated TLR4 expression and elevated levels of some DAMPs (HMGB1, S100A8, and S100A9) were observed in response to blood mixing. However, this action did not involve SAA and LPS-binding protein and did not lead to downstream proinflammatory cytokine (e.g., IL-6, TNF- $\alpha$, and IL-1 $\beta$ ) release after blood mixing, as shown in Figure 4 [8]. These findings may be explained as follows: First, LPS-binding proteins, which are soluble, are synthesized by hepatocytes, present in blood [21], and may not be synthesized in ex vivo studies or detected through ELISA. Second, negative regulators target several TLR4 signaling levels, and various molecules, such as SIGIRR and RP105, impede the initiation of this signaling cascade [8]. Other factors target molecules further downstream of TLR4 signaling through diverse processes [8]. Third, these results may have been obtained because of the short duration of blood mixing $(30 \mathrm{~min})$, during which time proinflammatory cytokines were not expressed. Therefore, the interaction between platelet TLR4 and certain DAMPs may not have triggered the proinflammatory cytokine release signaling pathway. Fourth, ex vivo blood mixing could not provide sufficient context in which to determine the full range of platelet responses to transfusion in vivo. Although our results suggest that platelet TLR4 functions as a pathophysiological link between innate immunity and transfusion, why the levels of proinflammatory cytokines downstream of the TLR4 signaling pathway were not increased remains an unanswered question.

Groups M, S, and I did not differ significantly in terms of TLR4 level. The major difference between them was the responses of antibodies recognizing transfusion antigens [22]. In most blood transfusions for humans, red blood cell membranes containing components of the ABO system are crucial features. These antibodies are implicated in the severest transfusion reactions. However, platelets do not have ABO systems [22]. Additionally, surface antigens on red blood cells include Duffy, Kell, Kidd, MNS, and P systems, which are not present on platelets [22]. We revealed no intergroup differences in platelet TLR4 expression, implying that reactions between antigens and antibodies had no role in TLR4 expression induction.

TLR4 ligands recognize DAMPs and pathogen-associated molecular patterns (PAMPs) $[5,14,15,23]$, such as circulating LPS (endotoxin). PAMPs are associated with microbial pathogens [24]. PAMP signaling is unlikely to participate proinflammatory cytokine release signaling pathway for the following two reasons: First, during blood mixing, PAMPs such as circulating LPS from microbial pathogens were not considered because no patients had a pathogenic infection. Second, after blood mixing, the levels of LPS-binding protein, which binds to PAMPs but not DAMPs, were not increased, as shown in Figure $4[14,15]$. Therefore, blood mixing may initiate platelet TLR4 expression, which could trigger an innate immune system response that is likely independent of PAMPs.

The rationale for selecting patients undergoing cardiac surgery in this study was as follows: First, blood transfusion is routinely required during cardiac surgery, and preparation for such surgery routinely requires cross-match testing of blood at least 1 day before the surgery. Therefore, cross-matched red blood cells were available, and they were sent to the operation room from the blood bank before anesthesia, enabling us to obtain donor cells. The cells were also used for heart-lung machine priming or stored until needed 
for transfusion. Second, patients undergoing cardiac surgery require arterial catheterization for aggressive hemodynamic monitoring. The arterial catheterization approach used for recipient blood sampling before anesthesia and skin incision prevents contamination from anesthetics and tissue damage. The anesthetics and tissue damage may be a confounding factor affecting platelet function and activation (Supplemental Figure S2). Red blood cells from groups $S$ and I were also obtained from the blood bank for other major surgeries at the study hospital.

Our blood mixing procedure is clinically relevant. We mixed, ex vivo, the donor and recipient blood; this method is similar to the cross-matching approach. Before a blood transfusion, cross-matching is applied to reveal the compatibility of a donor's blood with that of the recipient. The participants of this study had a mean body weight of $66.8 \mathrm{~kg}$; therefore, their total blood volume was around $4620 \mathrm{~mL}$ (approximately $7 \%$ of body weight). Our 1-10\% blood mixtures thus corresponded to volumes of around $46.8-467.6 \mathrm{~mL}$, respectively, which are commonly employed when administering transfusions.

This study had two limitations. First, the baseline levels of HMGB1, S100A8, S100A9, SAA, LPS-binding protein, IL-6, TNF- $\alpha$, and IL-1 $\beta$ were undetected in the donor red blood cells. However, these cells were added to the recipient whole blood at various volume concentrations $(1,5$, or $10 \%(v / v))$. The donor red blood cells had $55-60 \%$ hematocrit and these percentages were standardized. Therefore, they contained less plasma than the recipient whole blood did. Therefore, the plasma volume of our donor red blood cells was substantially smaller than that of our recipient whole blood. Second, we primarily used an ex vivo model because we included a group I (ABO-incompatible blood mixing) protocol, which could be harmful to patients if conducted in vivo. Therefore, in vivo host innate immune responses were not evaluated. Platelets have direct involvement in immune defense, and they assist with and regulate various activities of innate immune cells. Furthermore, platelets modulate immune cell function by attaching themselves to immune cells or releasing nucleosides, platelet-derived microvesicles, mitochondrial DNA, growth factors, lipid mediators, chemokines, and cytokines. Platelets and their associated releasates exert diverse effects on migration, microbicidal activity, differentiation, phagocytosis, clearance of pathogens, cytokine responses of innate immune cells, and extracellular trap formation [24].

\section{Conclusions}

Platelet TLR4 has actions at the crossroads of innate immune responses and thrombosis. We revealed that allogeneic blood mixing may modulate innate immune responses by upregulating platelet TLR4 and DAMPs, including HMGB1, S100A8, and S100A9, which may bind to TLR4, suggesting that platelet TLR4 links between transfusion and innate immunity in blood-mixing reactions. Given the numerous platelets that circulate in the blood, potential interactions between platelet TLR4 and DAMPs and an innate immune response is induced, leading to transfusion reactions, are possible. However, because TLR4 downstream proinflammatory cytokines were undetected, it remains unclear whether TLR4 signaling leads to a transfusion reaction under in vivo conditions. TLR4 expression upregulation was not associated with $\mathrm{ABO}$ compatibility. These findings indicate that TLR4 contributes to transfusion reactions that are unrelated to antibodies against red blood cell antigens. Whether platelet TLR4 is a novel therapeutic and prophylactic target in transfusion reactions or a new target for modulating innate immunity requires further study.

Supplementary Materials: The following are available online at https:/ / www.mdpi.com/article/10 .3390/biomedicines10010029/s1. Figure S1: Flow cytometry for platelet TLR4 expression, Figure S2: Key steps of general anesthesia and cardiac surgery performed.

Author Contributions: C.-S.T. and G.-S.H. conception and design of the work; C.-S.T. and G.-S.H. designed and executed the experiments; C.-S.T., M.-H.H., Y.-C.H. and G.-S.H. provided acquisition, analysis, and interpretation of data; C.-S.T. and G.-S.H. wrote the manuscript; C.-S.T., M.-H.H., Y.-C.H. 
and G.-S.H. reviewed, edited and approved the manuscript. All authors have read and agreed to the published version of the manuscript.

Funding: This study was supported by a grant from the Tri-Service General Hospital, Taipei, Taiwan, Grant no.: TSGH-C104-105, TSGH-C105-101, and TSGH-C106-085; and the Ministry of Science and Technology, Taipei, Taiwan, Grant no. MOST 107-2314-B-016-016.

Institutional Review Board Statement: The study was conducted according to the guidelines of the Declaration of Helsinki, and approved by the institutional review board of Tri-Service General hospital (TSGHIRB 1-102-05-014, TSGHIRB 1-107-05-015).

Informed Consent Statement: Informed consent was obtained from all subjects involved in the study.

Data Availability Statement: http:/ / www.chictr.org.cn/showproj.aspx?proj=123019. Accessed date: 22 November 2021.

Acknowledgments: The authors acknowledge the technical services (support) provided by the Instrument Center of the National Defense Medical Center.

Conflicts of Interest: The authors have no conflicts of interest or financial ties to disclose.

Clinical Trial Registration: The trial was registered at Chinese Clinical Trial Registry (ChiCTR2100045606). Accessed date: 22 November 2021.

\section{References}

1. Kheiri, B.; Abdalla, A.; Osman, M.; Haykal, T.; Chintalapati, S.; Cranford, J.; Sotzen, J.; Gwinn, M.; Ahmed, S.; Hassan, M.; et al. Restrictive versus liberal red blood cell transfusion for cardiac surgery: A systematic review and meta-analysis of randomized controlled trials. J Thromb. Thrombolysis 2019, 47, 179-185. [CrossRef] [PubMed]

2. Schattner, M. Platelet TLR4 at the crossroads of thrombosis and the innate immune response. J. Leukoc. Biol. 2019, 105, 873-880. [CrossRef] [PubMed]

3. Chukkapalli, S.S.; Ambadapadi, S.; Varkoly, K.; Jiron, J.; Aguirre, J.I.; Bhattacharyya, I.; Morel, L.M.; Lucas, A.R.; Kesavalu, L. Impaired innate immune signaling due to combined Toll-like receptor 2 and 4 deficiency affects both periodontitis and atherosclerosis in response to polybacterial infection. Pathog. Dis. 2018, 76, fty076. [CrossRef] [PubMed]

4. Cen, X.; Liu, S.; Cheng, K. The Role of Toll-Like Receptor in Inflammation and Tumor Immunity. Front. Pharmacol. 2018, 9, 878. [CrossRef]

5. D'Atri, L.P.; Schattner, M. Platelet toll-like receptors in thromboinflammation. Front. Biosci. 2017, 22, 1867-1883. [CrossRef]

6. Koupenova, M.; Clancy, L.; Corkrey, H.A.; Freedman, J.E. Circulating Platelets as Mediators of Immunity, Inflammation, and Thrombosis. Circ. Res. 2018, 122, 337-351. [CrossRef] [PubMed]

7. Ebermeyer, T.; Cognasse, F.; Berthelot, P.; Mismetti, P.; Garraud, O.; Hamzeh-Cognasse, H. Platelet Innate Immune Receptors and TLRs: A Double-Edged Sword. Int. J. Mol. Sci. 2021, 22, 7894. [CrossRef]

8. Lu, Y.C.; Yeh, W.C.; Ohashi, P.S. LPS/TLR4 signal transduction pathway. Cytokine 2008, 42, 145-151. [CrossRef]

9. Bosch, Y.P.; Al Dieri, R.; ten Cate, H.; Nelemans, P.J.; Bloemen, S.; de Laat, B.; Hemker, C.; Weerwind, P.W.; Maessen, J.G.; Mochtar, B. Measurement of thrombin generation intra-operatively and its association with bleeding tendency after cardiac surgery. Thromb. Res. 2014, 133, 488-494. [CrossRef]

10. Hermann, J.K.; Lin, S.; Soffer, A.; Wong, C.; Srivastava, V.; Chang, J.; Sunil, S.; Sudhakar, S.; Tomaszewski, W.H.; Protasiewicz, G.; et al. The Role of Toll-Like Receptor 2 and 4 Innate Immunity Pathways in Intracortical Microelectrode-Induced Neuroinflammation. Front. Bioeng. Biotechnol. 2018, 6, 113. [CrossRef]

11. Collao, N.; Rada, I.; Francaux, M.; Deldicque, L.; Zbinden-Foncea, H. Anti-Inflammatory Effect of Exercise Mediated by Toll-Like Receptor Regulation in Innate Immune Cells-A Review. Int. Rev. Immunol. 2020, 39, 39-52. [CrossRef] [PubMed]

12. Tantawy, H.; Li, A.; Dai, F.; Elgammal, M.; Sukumar, N.; Elefteriades, J.; Akhtar, S. Association of red blood cell transfusion and short- and longer-term mortality after coronary artery bypass graft surgery. J. Cardiothorac. Vasc. Anesth. 2018, 32, 1225-1232. [CrossRef] [PubMed]

13. Sun, S.; He, M.; Wang, Y.; Yang, H.; Al-Abed, Y. Folic acid derived-P5779 mimetics regulate DAMP-mediated inflammation through disruption of HMGB1:TLR4:MD-2 axes. PLoS ONE 2018, 13, e0193028. [CrossRef] [PubMed]

14. Cognasse, F.; Laradi, S.; Berthelot, P.; Bourlet, T.; Marotte, H.; Mismetti, P.; Garraud, O.; Hamzeh-Cognasse, H. Platelet Inflammatory Response to Stress. Front. Immunol. 2019, 10, 1478. [CrossRef] [PubMed]

15. Wang, P.; Tian, H.; Zhang, Z.; Wang, Z. EZH2 Regulates Lipopolysaccharide-Induced Periodontal Ligament Stem Cell Proliferation and Osteogenesis through TLR4/MyD88/NF-kappaB Pathway. Stem Cells Int. 2021, 2021, 7625134. [CrossRef] [PubMed]

16. Zettel, K.R.; Dyer, M.; Raval, J.S.; Wu, X.; Klune, J.R.; Gutierrez, A.; Triulzi, D.J.; Billiar, T.R.; Neal, M.D. Aged Human Stored Red Blood Cell Supernatant Inhibits Macrophage Phagocytosis in an HMGB1 Dependent Manner after Trauma in a Murine Model. Shock 2017, 47, 217-224. [CrossRef] 
17. Ottestad, W.; Rognes, I.N.; Skaga, E.; Frisvoll, C.; Haraldsen, G.; Eken, T.; Lundback, P. HMGB1 concentration measurements in trauma patients: Assessment of pre-analytical conditions and sample material. Mol. Med. 2019, 26, 5. [CrossRef]

18. Miller, R.D. Patient Blood Management: Transfusion Therapy, Miller's Anesthesia, 9th ed.; Elsevier: Philadelphia, PA, USA, 2020; Chapter 49; pp. 1546-1576.

19. Qing, D.Y.; Conegliano, D.; Shashaty, M.G.; Seo, J.; Reilly, J.P.; Worthen, G.S.; Huh, D.; Meyer, N.J.; Mangalmurti, N.S. Red blood cells induce necroptosis of lung endothelial cells and increase susceptibility to lung inflammation. Am. J. Respir. Crit. Care Med. 2014, 190, 1243-1254. [CrossRef]

20. Islam, N.; Whitehouse, M.; Mehendale, S.; Hall, M.; Tierney, J.; O’Connell, E.; Blom, A.; Bannister, G.; Hinde, J.; Ceredig, R.; et al. Post-traumatic immunosuppression is reversed by anti-coagulated salvaged blood transfusion: Deductions from studying immune status after knee arthroplasty. Clin. Exp. Immunol. 2014, 177, 509-520. [CrossRef]

21. Vallance, T.M.; Zeuner, M.T.; Williams, H.F.; Widera, D.; Vaiyapuri, S. Toll-Like Receptor 4 Signalling and Its Impact on Platelet Function, Thrombosis, and Haemostasis. Mediat. Inflamm. 2017, 2017, 9605894. [CrossRef]

22. Thornton, N.M.; Grimsley, S.P. Clinical significance of antibodies to antigens in the ABO, MNS, P1PK, Rh, Lutheran, Kell, Lewis, Duffy, Kidd, Diego, Yt, and Xg blood group systems. Immunohematology 2019, 35, 95-101. [CrossRef] [PubMed]

23. Wu, K.; Zhang, H.; Fu, Y.; Zhu, Y.; Kong, L.; Chen, L.; Zhao, F.; Yu, L.; Chen, X. TLR4/MyD88 signaling determines the metastatic potential of breast cancer cells. Mol. Med. Rep. 2018, 18, 3411-3420. [CrossRef] [PubMed]

24. Ribeiro, L.S.; Migliari Branco, L.; Franklin, B.S. Regulation of Innate Immune Responses by Platelets. Front. Immunol. 2019, 10, 1320. [CrossRef] [PubMed] 